\title{
Rapid identification of novel independent serum biomarkers in diffuse large B-cell lymphoma
}

\author{
Yi-Qun Che ${ }^{1 \#}$, Di Wang ${ }^{1 \#}$, Peng Liu ${ }^{2}$, Yue Zhang ${ }^{1}$, Yang Luo ${ }^{2}$, Hui-Ying Liu ${ }^{3}$, Di Shen ${ }^{1}$, Wei Cui ${ }^{1}$ \\ ${ }^{1}$ Department of Clinical Laboratory, ${ }^{2}$ Department of Medical Oncology, National Cancer Center/National Clinical Research Center for Cancer/ \\ Cancer Hospital, Chinese Academy of Medical Sciences and Peking Union Medical College, Beijing, China; ${ }^{3}$ Department of Clinical Laboratory, \\ Jinzhou Central Hospital, Jinzhou, China \\ Contributions: (I) Conception and design: YQ Che, W Cui; (II) Administrative support: YQ Che, W Cui; (III) Provision of study materials or patients: \\ P Liu, Y Luo; (IV) Collection and assembly of data: HY Liu, D Shen; (V) Data analysis and interpretation: D Wang, Y Zhang; (VI) Manuscript \\ writing: All authors; (VII) Final approval of manuscript: All authors. \\ \#These authors contributed equally to this work. \\ Correspondence to: Wei Cui. Department of Clinical Laboratory, National Cancer Center/National Clinical Research Center for Cancer/Cancer \\ Hospital, Chinese Academy of Medical Sciences and Peking Union Medical College, 17 Panjiayuan Nanli, Chaoyang District, Beijing, China. \\ Email: wendycuiwei@sina.cn.
}

Background: Diffuse large B-cell lymphoma (DLBCL) is a common subtype of non-Hodgkin's lymphoma (NHL). We assessed serum biomarkers to identify patients with DLBCL and healthy controls, as well as prognosis-related paired pre- and post-R-CHOP therapy effect of patients with DLBCL.

Methods: Serum samples from 329 DLBCL patients and 100 healthy controls were collected in this study, as well as 72 samples from additional DLBCL patients with paired pre- and post-R-CHOP therapy $(n=36)$. All serum samples were pretreated and detected by matrix-assisted laser desorption ionization time-of-flight mass spectrometry (MALDI-TOF-MS). Serum biomarkers were discriminated between DLBCL patients and healthy controls, as well as between DLBCL patients with prognosis-related paired pre- and post-R-CHOP therapy, and identified by Veristrat Identification Platform 1.0, Bioyong Explore 1.0 and mMass software.

Results: Two peaks, $\mathrm{m} / \mathrm{z}$ values of $15,140.74$ and $15,885.43$, were significantly different from all peaks among the DLBCL patients and healthy controls $(\mathrm{P}<0.001)$. Patients with DLBCL could be identified with a sensitivity of $85.9 \%$ and specificity of $91.8 \%$ of $15,140.74$, in the same way for the sensitivity of $92.1 \%$ and specificity of $84.4 \%$ of $15,885.43$. The $\mathrm{m} / \mathrm{z}$ values of $13,895.80$ were downregulated after R-CHOP treatment in DLBCL patients who obtained complete remission (CR) $(\mathrm{P}=0.018)$.

Conclusions: The potential independent serum biomarkers for diagnosis as well as prognosis of DLBCL could be provided for rapid identification.

Keywords: Matrix-assisted laser desorption ionization time-of-flight mass spectrometry (MALDI-TOF-MS); diffuse large B-cell lymphoma (DLBCL); diagnosis; therapy monitoring; biomarker

Submitted Dec 14, 2019. Accepted for publication Jun 28, 2020.

doi: $10.21037 /$ tcr-19-2809

View this article at: http://dx.doi.org/10.21037/tcr-19-2809

\section{Introduction}

Non-Hodgkin's lymphoma (NHL) is a member of the top 10 causes of cancer mortality (1). Diffuse large B-cell lymphoma (DLBCL) is the most common NHL, accounting for approximately $30-40 \%$ of all NHL. Mortality of DLBCL increased in China (2). Early DLBCL often have no detectable signs. DLBCL is a group of malignant tumors with considerable heterogeneity in clinical manifestations, histomorphology, and prognosis (3). DLBCL can take place in any part of the body or any organ and the clinical manifestation is an invasive course (4). The current "gold standard" for the diagnosis of DLBCL is still histopathology (5). 
Due to the extreme heterogeneity, DLBCL pathological structure is so complex that immunohistochemistry must be added to make the diagnosis clear. Core needle aspiration biopsy is extensively used because lymph node painless enlargement is the primary symptom mostly of DLBCL, but the biopsy is a traumatic examination. The patients' acceptance is limited and it is difficult to promote it among the population. In addition, a few DLBCL appears to be insidious, with no superficial lymph nodes. Some DLBCL originating from the mediastinum, abdominal cavity, and other external sites are relatively tough to obtain samples. What is said above should bring great difficulties to pathological diagnosis. LDH, $\beta 2-\mathrm{MG}$, and CA125 are common blood markers of NHL. Studies have shown that $\mathrm{LDH}$ level reflects tumor burden, and elevated $\mathrm{LDH}$ and $32-M G$ levels are independent prognostic factors for DLBCL. The level of CA125 expression is closely related to the malignant degree and progression-free survival of lymphoma patients. However, these indicators have poor sensitivity and specificity in the diagnosis of DLBCL (6-8). Currently, those serum tumor markers are not commonly used in clinical practice. It is urgently required to look for innovative, reliable, effective and noninvasive tumor biomarkers between DLBCL patients and healthy controls.

After the emergence of rituximab, the R-CHOP regimen significantly improved overall survival and progression-free survival in patients, and about $50 \%$ of patients were cured by chemotherapy, radiation therapy, and immunotherapy. However, $30 \%$ of patients still develop into relapsed/ refractory DLBCL after initial treatment, who have only $10 \%$ chance of cure even with high-dose chemotherapy combined with autologous hematopoietic stem cell transplantation $(9,10)$. Otherwise, DLBCL resistance occurred more than half a year after R-CHOP treatment. The primary lesions had less margin or the compliance of patients with new lesions was poor. Then biopsy was hardly to taken again. The detection of tumor micro-residue (MRD) and the identification of tumor recurrence after treatment have great limitations. Therefore, noninvasive early diagnosis biomarkers and accompanying efficacy factors of the treatment and prognosis of DLBCL patients are extremely important.

Human serum contains complex peptides, some of which may be involved in disease concerning their relative abundance or presence and are useful as biomarkers for diagnosis or prognosis (11). Matrix-assisted laser desorption ionization (MALDI) time-of-flight (TOF) mass spectrometry (MS) is a fast and sensitive technique for analyzing complex biological samples such as serum, urine, and tissue. It takes laser pulses to ionize the analytes on the chip, calculate the flight time of ions with different mass-to-charge ratios in the electric field, draw them into different mass spectra, and then analyze them by computer software to display various information of the proteins (12). In this study, we utilized MALDI-TOF-MS to analyze serum samples from DLBCL patients and healthy controls, as well as DLBCL patients who underwent R-CHOP treatment. Then the potential biomarkers closely associated with tumorigenesis were identified by MALDI-TOF- MS. We presented the following article in accordance with the STARD reporting checklist (available at http://dx.doi. org/10.21037/tcr-19-2809).

\section{Methods}

\section{Patients}

Three hundred and twenty-nine serum samples were collected from patients with newly diagnosed DLBCL who were confirmed according to their histopathological examination results at National Cancer Center/Cancer Hospital of Chinese Academy of Medical Sciences between October 2017 and March 2018. There were 164 males, aged 15-84 years, with a median age of 49 years and 165 females, aged 9-77 years, with a median age of 54 years in this retrospective study. In addition, paired serum samples from 32 patients with pre- and post-R-CHOP therapy were collected. All patients underwent R-CHOP six-cycle chemotherapy and were followed for more than one year. There were 30 males and 2 females, and 16 patients who achieved complete remission (CR) during follow-up, and 16 patients with partial remission. The serum of 100 healthy controls was collected, including 46 males and 54 females, aged 21-69 years.

The study was conducted in accordance with the Declaration of Helsinki (as revised in 2013). The study was approved by Ethics Committee of Cancer Hospital, Chinese Academy of Medical Sciences (Beijing, China) (No. NCC2015B-01) and informed consent was taken from all the patients.

\section{Serum collection}

Venous blood samples $(<5 \mathrm{~mL})$ were collected into serum collection tubes for clinical routine biochemistry tests, the rest serum samples $(150 \mu \mathrm{L})$ after detection were 
left at room temperature for about $1 \mathrm{~h}$ before being centrifuged at $820 \mathrm{~g}$ for $10 \mathrm{~min}$ at $4{ }^{\circ} \mathrm{C}$. The resulting serum was transferred into new tubes, followed by further centrifugation at $16,000 \mathrm{~g}$ for $10 \mathrm{~min}$ at $4^{\circ} \mathrm{C}$, to completely remove any cell debris. Afterward, serum samples were transferred to the new tubes and stored at $-80{ }^{\circ} \mathrm{C}$.

\section{MALDI-TOF MS analysis}

$500 \mu \mathrm{L}$ of acetonitrile (Wako), $499 \mu \mathrm{L}$ of ultrapure water, and $1 \mu \mathrm{L}$ of trifluoroacetic acid (Sigma) were thoroughly mixed to prepare a mixed solution. $25 \mathrm{mg}$ of Sinapic Acid (SA) matrix powder (Sigma) was weighed into the above mixture. After vortexing for $2 \mathrm{~min}$, ultrasonication for $5 \mathrm{~min}$, a SA matrix solution was obtained. Serum samples were taken from $-80{ }^{\circ} \mathrm{C}$ and thawed at $4{ }^{\circ} \mathrm{C}$. Each sample was diluted 1:10 with HPLC grade water and the dilutions were vortexed for $30 \mathrm{~s}$. The diluted sample was then mixed 1:1 with the SA substrate solution as described above and vortexed for $30 \mathrm{~s}$. Each sample matrix mixture was taken at $1.5 \mu \mathrm{L}$ for spotting and air-dried at room temperature for MALDI-TOF-MS (Clin-TOF-II, Bioyong Inc., Beijing, China) detection. The Veristrat identification platform 1.0 was used to group the mass spectral data of DLBCL patients, and the gel maps of DLBCL and healthy controls were plotted by Bioyong Explorer 1.0 software, and the mass spectral data of lymphoma and healthy controls were analyzed by mMass software.

\section{Repeatability and stability testing}

Each serum sample was repeatedly tested 3 times and the median was taken as the result. Moreover, serum samples from 20 patients were randomly selected and detected twice by MALDI-TOF-MS. The mass spectra of protein expressions in 20 serum samples were compared and the consistency of the detected values was analyzed.

\section{Statistical analysis}

Statistical analysis was conducted using SPSS 22.0 statistical software. After the normality test, the data with skewed distribution were expressed by the median (interquartile range), and the data of each group were compared using the nonparametric test. The data with normal distribution were expressed by mean \pm standard deviation, and the data of each group were compared by $t$-test. The diagnostic performance of each index was compared using the receiver operating characteristic curve (ROC) and the area under the curve (AUC). The difference between the groups was statistically significant at $\mathrm{P}<0.05$.

\section{Results}

\section{Mass spectrometry generation of DLBCL and bealthy control and assay reproducibility}

A gel map of mass spectrometry data of DLBCL patients and healthy human was generated by Bioyong Explorer 1.0 software. It was found that DLBCL patients (red) had two distinct mass spectral peaks at 15,000-16,250 (m/z) compared to healthy subjects (green) (Figure 1A). Twenty randomly selected serum samples were tested twice with a 2-week interval for consistency analysis and found a correlation coefficient of 0.9574 . Thus, it suggested that this experiment is helpful in detecting peptide repeatability in serum samples of DLBCL patients and the results are stable (Figure 1B).

\section{Serum differential proteins of DLBCL patients and bealthy controls}

Peak analysis of the differential peaks in the gel maps of DLBCL patients and healthy subjects using mMass software revealed that the two peaks (m/z values: $15,140.74$ and $15,885.43)$ were significantly elevated in DLBCL compared to healthy individuals $(\mathrm{P}<0.001)$ (Table 1). Using these two differential peaks to diagnose 329 DLBCL patients and 100 healthy people, the AUC of peak 1 was 0.954 with $85.9 \%$ sensitivity and $91.8 \%$ specificity. The AUC of peak 2 was 0.956 and the sensitivity and specificity were $92.1 \%$ and 84.4\% respectively (Figure 2 \& Table 2).

\section{The relationship between mass spectrometry peaks and prognosis of DLBCL patients}

Thirty-two patients with DLBCL who were enrolled in matched serum were treated with 6 cycles of R-CHOP and had at least one year of complete follow-up data. Among these patients, 16 achieved CR (green) and 16 had no CR (red). These DLBCL patients were grouped according to two mass spectral peaks (m/z values: 11,685 and 11,759$)$ in the Veristrat identification platform, and the two groups of DLBCL patients with different prognosis were separated (Figure $3 A$ ). To observe the mass spectral peaks intensity of serum samples with pre- and post-R-CHOP treatment, 
A

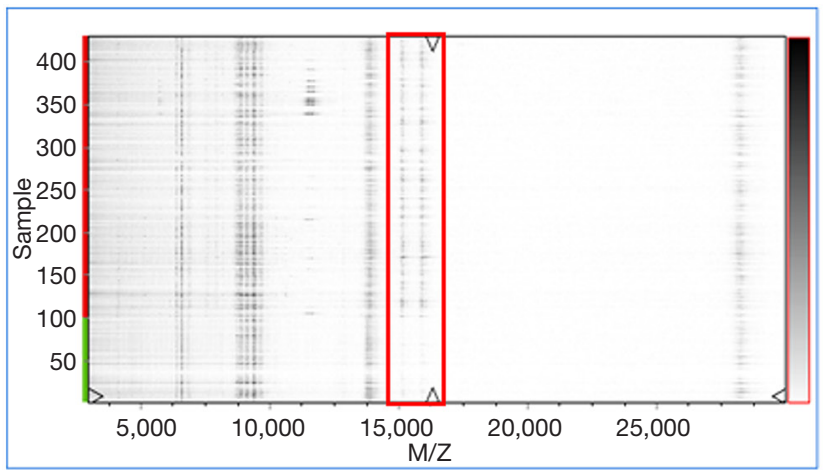

B

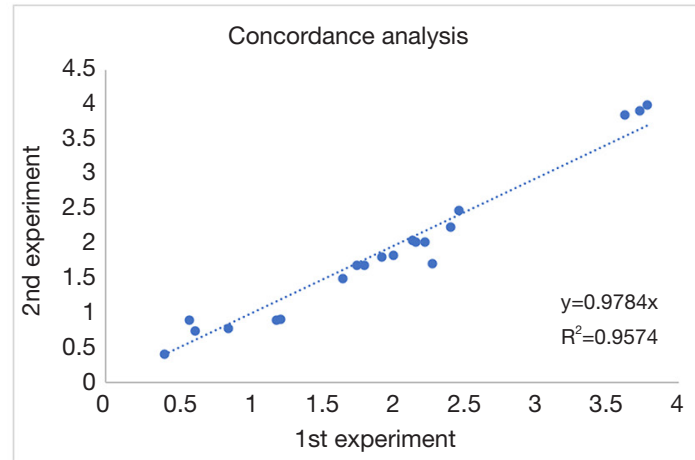

Figure 1 Mass spectrometry generation of diffuse large B-cell lymphoma (DLBCL) and healthy control and assay reproducibility. Mass spectrometry gel map of diffuse large B-cell lymphoma (DLBCL) patients (red) and healthy people (green) (A). Consistency analysis of the matrix-assisted laser desorption ionization time-of-flight mass spectrometry (MALDI-TOF MS) technique (B).

Table 1 Differential protein in diffuse large B-cell lymphoma (DLBCL) and healthy human serum by matrix-assisted laser desorption ionization time-of-flight mass spectrometry (MALDI-TOF MS)

\begin{tabular}{lccc}
\hline PEAK $(\mathrm{m} / \mathrm{z})$ & Cases & Median (interquartile range) & Mann-Whitney U \\
\hline $15,140.74$ & & $207.00(417.00)$ & $1,447.500$ \\
Healthy & 97 & $4,980.50(9,888.00)$ & \\
DLBCL & 326 & & $1,378.500$ \\
$15,885.43$ & 96 & $118.00(253.50)$ & $<0.001$ \\
Healthy & 328 & $4,556.00(8,888.25)$ & \\
DLBCL & 328 & \\
\hline
\end{tabular}
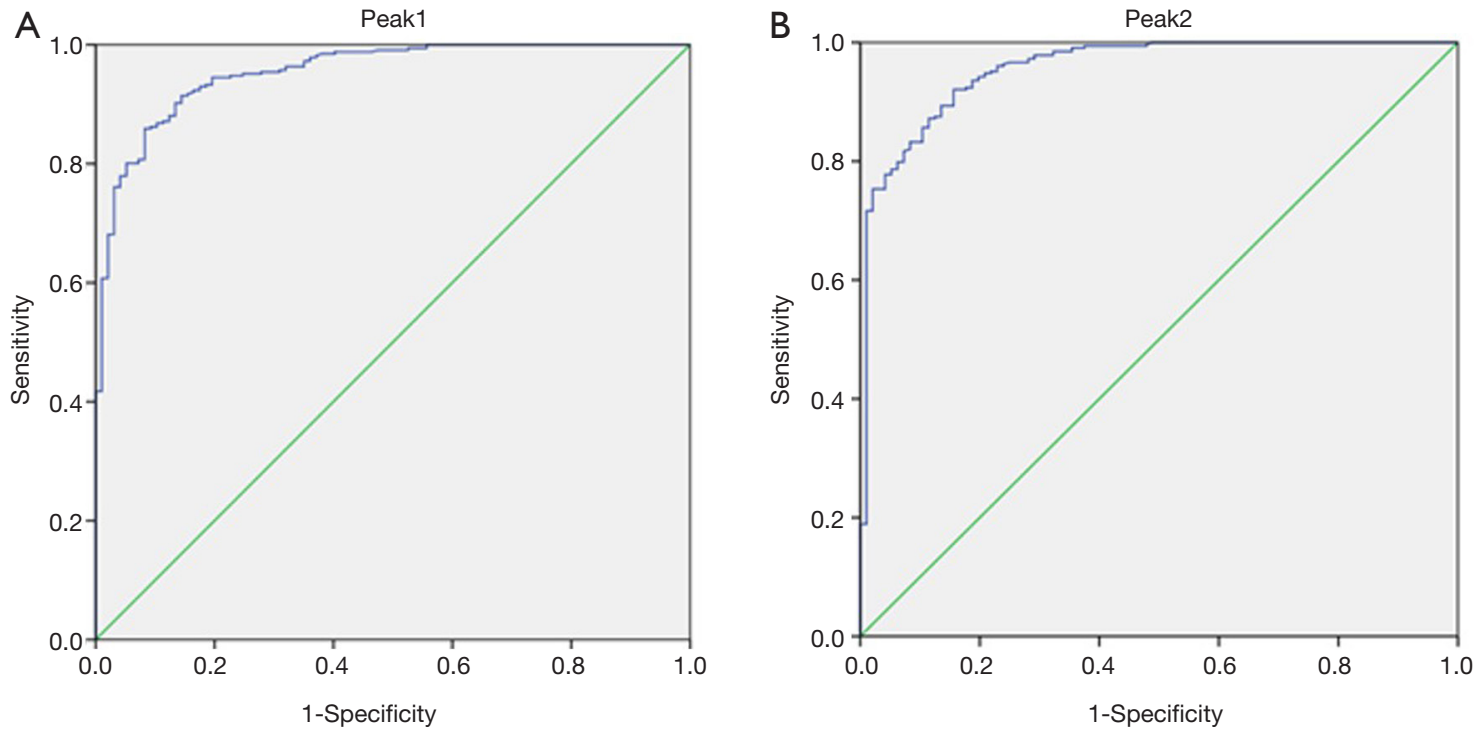

Figure 2 Receiver operating characteristic (ROC) curves for the two selected peaks. Peak1 (m/z): 15,140.74 (A); Peak2 (m/z): 15,885.43 (B). 
Table 2 Area under the curve (AUC) values of the two selected peaks

\begin{tabular}{lcccccc}
\hline Group $(\mathrm{m} / \mathrm{z})$ & DLBCL & Healthy & AUC & P & Sensitivity & Specificity \\
\hline Peak1: $15,140.74$ & 326 & 97 & 0.954 & $<0.001$ & $85.9 \%$ & $91.8 \%$ \\
Peak2: $15,885.43$ & 328 & 96 & 0.956 & $<0.001$ & $92.1 \%$ & $84.4 \%$ \\
\hline
\end{tabular}

DLBCL, diffuse large B-cell lymphoma.

we found that among the 16 patients who achieved CR, 9 patients had a significant decrease in the $\mathrm{m} / \mathrm{z}$ of $13,895.8$ after $\mathrm{R}-\mathrm{CHOP}$ treatment $(\mathrm{P}=0.018)$. Therefore, this phenomenon was not complied with patients who did not reach CR (Figure 3B, C \& Table 3).

\section{Discussion}

DLBCL is a malignant tumor derived from the lymphatic system. During its development, it will form a specific proteome, which constitutes the characteristic protein fingerprint pattern of the disease, namely serum protein biomarkers. MALDI-TOF-MS technique used in this study is a novel protein profiling technology developed in recent years, which becomes the main analytical technique of proteomics with its rapid, accurate and sensitive testing (13). It has been found that biomarkers obtained by MALDITOF-MS technology are mostly low molecular weight proteins produced by specific tumor microenvironments (14), and the sensitivity and specificity of this technology for early detection and early diagnosis of various solid tumors such as lung cancer, ovarian cancer, intestinal cancer, and esophageal cancer are more than $80 \%$, which are superior to traditional tumor markers (15-18). This technology can have important clinical application value in the early diagnosis of lymphoma. However, there are not many studies on DLBCL using MALDI-TOF-MS technology internationally. Most of the patients enrolled in studies were lymphoma patients who did not distinguish between pathological types, so the protein peaks obtained were different (19). There are numerous pathological types of lymphoma. Not only T-cell lymphoma and B-cell lymphoma are very different, but B-cell lymphoma is also divided into various subtypes with great heterogeneity (20). Therefore, the use of lymphoma as a population for mass spectrometry has serious limitations. This study focused on one pathological type-DLBCL, with more cases than commonly studied, and the results were more representative.

There is presently a lack of highly sensitive and specific serological biomarkers for early diagnosis of lymphoma. In this study, we utilized MALDI-TOF-MS to analyze serum protein profiles in 329 DLBCL patients and 100 healthy individuals and found two DLBCL-specific "marker proteins" (m/z 15,140.74 and 15,885.43). The AUC of peak 1 was 0.954 with $85.9 \%$ sensitivity and $91.8 \%$ specificity. The AUC of peak 2 was 0.956 and the sensitivity and specificity were $92.1 \%$ and $84.4 \%$ respectively. They highly expressed in lymphoma and had a good diagnostic performance.

Relapsed/refractory DLBCL has been an important problem plagued clinicians. If the efficacy of DLBCL is expected to be good or poor at the onset of pre-treatment, or if the patients' drug resistance is predicted by monitoring serum protein changes during R-CHOP treatment, we can give individualized treatment to the patients. Routine treatment is given to patients with mild predictive outcomes. Patients with poor efficacy will increase the intensity of treatment or prolong the treatment period according to the peculiar conditions of patients, and apply biological immunotherapy. This will help doctors choose the best treatment for each patient and achieve better outcome. Therefore, in addition to initial diagnosis, we also compared serum protein markers associated with DLBCL prognosis and treatment monitoring. In the study, we found that DLBCL patients with different prognosis were separated by two mass spectral peaks (m/z values: 11,685 and 11,759). Further analysis of mass spectral peaks intensity before and after R-CHOP therapy found that DLBCL patients after R-CHOP treatment with significantly decreased protein $(\mathrm{m} / \mathrm{z}$ values: $13,895.8)$ expression were the most likely to achieve CR. These results provide an adjunct to clinicians in predicting the prognosis of DLBCL patients. However, there were only 32 patients with DLBCL who had consistent treatment regimens and more than one year of complete prognostic data in this study.

In summary, this study screened the differential protein of DLBCL by MALDI-TOF-MS, which has superior diagnostic efficiency, high sensitivity, and high specificity, and is helpful for the preliminary screening diagnosis 
A

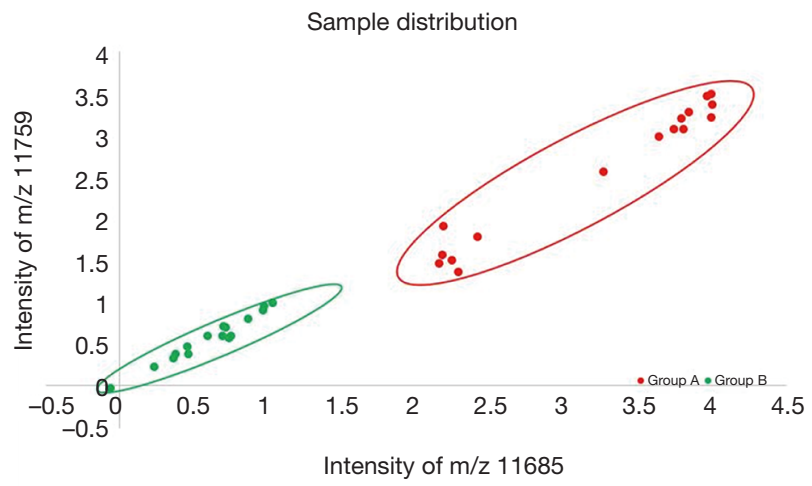

C.
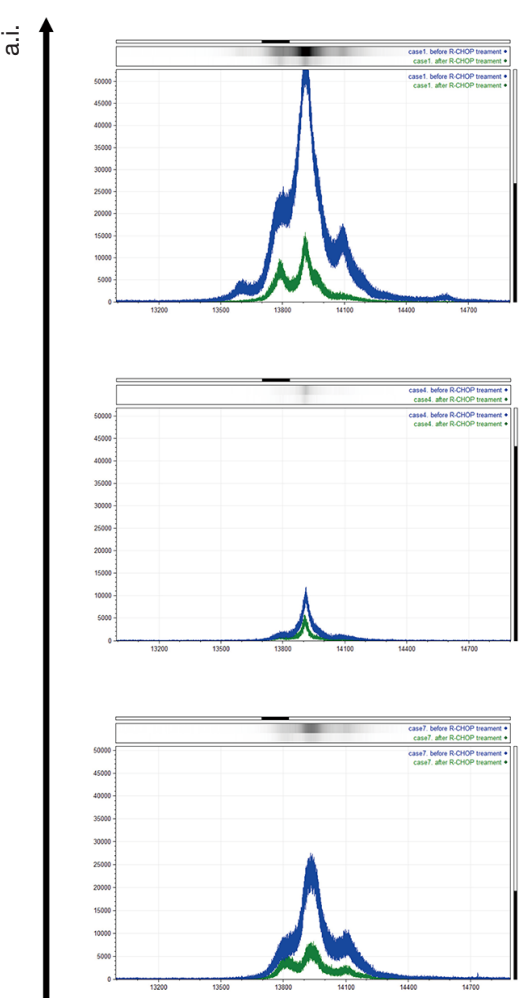
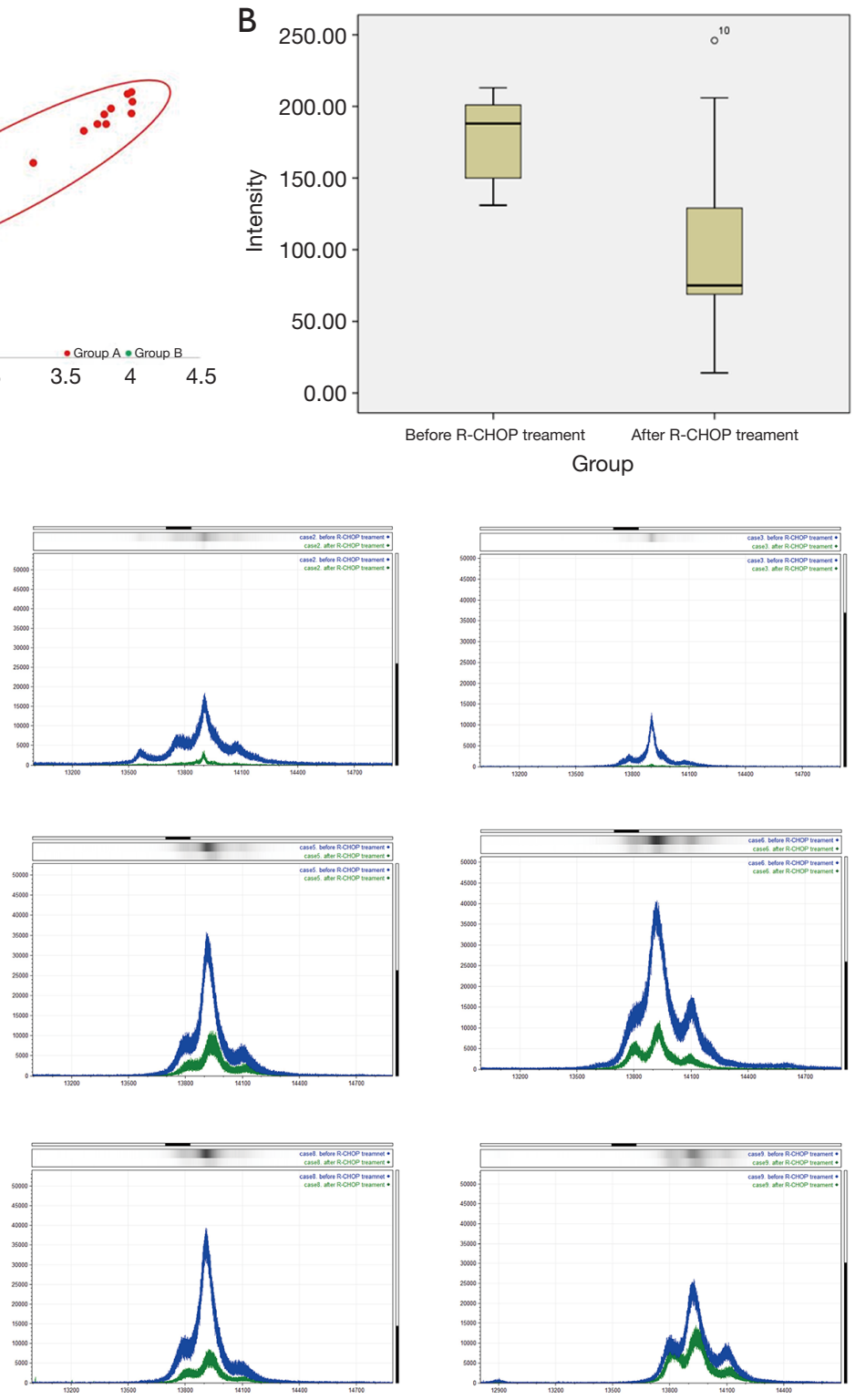

Figure 3 The distribution plot of group complete remission (CR) (green) vs. non-CR (red) (A). The mass spectral peaks intensity of nine diffuse large B-cell lymphoma (DLBCL) patients had a significant decrease in the m/z of 13,895.8 after R-CHOP (rituximab plus cyclophosphamide, doxorubicin, vincristine, and prednisone) (B\&C).

Table 3 The mass spectral peak of 13,895.80 before and after R-CHOP (rituximab plus cyclophosphamide, doxorubicin, vincristine, and prednisone) treatment in diffuse large B-cell lymphoma (DLBCL) patients achieving complete remission

\begin{tabular}{lllll}
\hline Peak $(\mathrm{m} / \mathrm{z})$ & Cases & $\bar{\chi} \pm \mathrm{s}$ & $\mathrm{T}$ & $\mathrm{P}$ \\
\hline Before R-CHOP treatment & 9 & $178.89 \pm 32.49$ & 2.646 & 0.018 \\
After R-CHOP treatment & 9 & $107.11 \pm 74.61$ & \\
\hline
\end{tabular}


of DLBCL. In addition, we analyzed differential serum protein peaks associated with different prognosis and $\mathrm{R}-\mathrm{CHOP}$ efficacy. Further increase in the number of cases analysis and identification is expected to provide valuable information for the identification, prognosis, and efficacy of DLBCL.

\section{Acknowledgments}

Funding: This work was supported by the National Natural Science Foundation of China (81972016) and Chinese Academy of Medical Sciences Innovation Fund for Medical Sciences (2017-I2M-3-005 \& 2017-I2M-1-013) \& Beijing Municipal Natural Science Foundation (7162164).

\section{Footnote}

Reporting Checklist: The authors have completed the STARD reporting checklist. Available at http://dx.doi.org/10.21037/ tcr-19-2809

Data Sharing Statement: Available at http://dx.doi. org/10.21037/tcr-19-2809

Conflicts of Interest: All authors have completed the ICMJE uniform disclosure form (available at http://dx.doi. org/10.21037/tcr-19-2809). The authors have no conflicts of interest to declare.

Ethical Statement: The authors are accountable for all aspects of the work in ensuring that questions related to the accuracy or integrity of any part of the work are appropriately investigated and resolved. The study was conducted in accordance with the Declaration of Helsinki (as revised in 2013). The study was approved by Ethics Committee of Cancer Hospital, Chinese Academy of Medical Sciences (Beijing, China) (No. NCC2015B-01) and informed consent was taken from all the patients.

Open Access Statement: This is an Open Access article distributed in accordance with the Creative Commons Attribution-NonCommercial-NoDerivs 4.0 International License (CC BY-NC-ND 4.0), which permits the noncommercial replication and distribution of the article with the strict proviso that no changes or edits are made and the original work is properly cited (including links to both the formal publication through the relevant DOI and the license). See: https://creativecommons.org/licenses/by-nc-nd/4.0/.

\section{References}

1. Siegel RL, Miller KD, Jemal A. Cancer statistics, 2019. CA Cancer J Clin 2019;69:7-34.

2. Liu W, Liu J, Song Y, et al. Mortality of lymphoma and myeloma in China, 2004-2017: an observational study. J Hematol Oncol 2019;12:22.

3. Al-Hamadani M, Habermann TM, Cerhan JR, et al. NonHodgkin lymphoma subtype distribution, geodemographic patterns, and survival in the US: a longitudinal analysis of the National Cancer Data Base from 1998 to 2011. Am J Hematol 2015;90:790-5.

4. Smith A, Howell D, Patmore R, et al. Incidence of haematological malignancy by sub-type: a report from the Haematological Malignancy Research Network. Br J Cancer 2011;105:1684-92.

5. Benboubker L, Valat C, Linassier C, et al. A new serologic index for low-grade non-Hodgkin's lymphoma based on initial CA125 and LDH serum levels. Ann Oncol 2000;11:1485-91.

6. Park JH, Yoon DH, Kim DY, et al. The highest prognostic impact of $\mathrm{LDH}$ among international prognostic indices (IPIs): an explorative study of five IPI factors among patients with DLBCL in the era of rituximab. Ann Hematol 2014;93:1755-64.

7. Ji H, Niu X, Yin L, et al. Ratio of immune response to tumor burden predicts survival via regulating functions of lymphocytes and monocytes in diffuse large B-cell lymphoma. Cell Physiol Biochem 2018;45:951-61.

8. Wu JZ, Tian T, Huang Y, et al. Serum carbohydrate antigen 125 concentration as a superior predictor for serosal effusion at diagnosis and a prognostic factor in diffuse large B-cell lymphoma. Cancer Biomark 2016;17:205-12.

9. Nowakowski GS, Czuczman MS. ABC, GCB, and doublehit diffuse large B-cell lymphoma: does subtype make a difference in therapy selection? Am Soc Clin Oncol Educ book 2015;35:e449-57.

10. Van Den Neste E, Schmitz N, Mounier N, et al. Outcomes of diffuse large B-cell lymphoma patients relapsing after autologous stem cell transplantation: an analysis of patients included in the CORAL study. Bone Marrow Transplant 2017;52:216-21.

11. Yang J, Yang J, Gao Y, et al. Identification of potential serum proteomic biomarkers for clear cell renal cell carcinoma. PLoS One 2014;9:e111364.

12. Hortin GL. The MALDI-TOF mass spectrometric view of the plasma proteome and peptidome. Clin Chem 
2006;52:1223-37.

13. Sahar T, Nigam A, Anjum S, et al. Interactome analysis of the differentially expressed proteins in uterine leiomyoma. Anticancer Agents Med Chem 2019;19:1293-312.

14. Stübiger G, Nairn MD, Abban TK, et al. MALDI-MS protein profiling of chemoresistance in extracellular vesicles of cancer cells. Anal Chem 2018;90:13178-82.

15. Klupczynska A, Swiatly A, Hajduk J, et al. Identification of serum peptidome signatures of non-small cell lung cancer. Int J Mol Sci 2016;17:410.

16. Deng BG, Yao JH, Liu QY, et al. Comparative serum proteomic analysis of serum diagnosis proteins of colorectal cancer based on magnetic bead separation and MALDI-TOF mass spectrometry. Asian Pac J Cancer Prev 2013;14:6069-75.

17. Swiatly A, Horala A, Hajduk J, et al. MALDI-TOF-MS

Cite this article as: Che YQ, Wang D, Liu P, Zhang Y, Luo Y, Liu HY, Shen D, Cui W. Rapid identification of novel independent serum biomarkers in diffuse large B-cell lymphoma. Transl Cancer Res 2020;9(8):4534-4541. doi: 10.21037/ tcr-19-2809 analysis in discovery and identification of serum proteomic patterns of ovarian cancer. BMC Cancer 2017;17:472.

18. Zaki A, Ramadan RA, Moez P, et al. Plasma peptidome pattern of breast cancer using magnetic beads-based plasma fractionation and MALDI-TOF MS: a case control study in Egypt. Asian Pac J Cancer Prev 2019;20:175-84.

19. Ueda K, Nakanishi T, Shimizu A, et al. Identification of L-plastin autoantibody in plasma of patients with nonHodgkin's lymphoma using a proteomics-based analysis. Ann Clin Biochem 2008;45:65-9.

20. National Comprehensive Cancer Network. (NCCN) Clinical Practice Guidelines in Oncology. B-Cell Lymphomas, Version 1. 2019. Available online: https:// www.nccn.org/professionals/physician_gls/pdf/b-cell_ blocks.pdf. Accessed: 3 Mar 2019. 